\title{
Histopathology of vasculitis
}

\author{
G. Bajocchi' ${ }^{1}$ A. Cavazza ${ }^{2}$ \\ ${ }^{1}$ Rheumatology Uni, S. Maria Hospital - USL, IRCCS Institute, Reggio Emilia, Italy; \\ ${ }^{2}$ Pathology Unit., S. Maria Hospital - USL, IRCCS Institute, Reggio Emilia, Italy
}

\begin{abstract}
SUMMARY
The range of pathologies that are related to primitive vasculitis is broad, complex and not as typical as we would expect. Clinicians should be aware that several forms of primitive and systemic vasculitis, regardless of the size of the affected vessel, may exhibit identical histological alterations. This observation has important clinical implications as it means that cases of vasculitis do not correspond clinically and histologically. Thus, while histology remains the diagnostic gold standard, it can be used only as part of the most complete clinical assessment possible.

Another point worth of the clinician's attention is that vasculitis histology changes over time, as do disease evolution and activity, even without considering the masking effects of treatment and the possibility of sampling error due to the patchy occurrence of vasculitis.

The purpose of this review is to identify the most common forms of vasculitis in clinical practice, and to provide guidance to the clinician on the pathology of the vessels.
\end{abstract}

Key words: Vasculitis; Histopathology.

Reumatismo, 2018; 70 (3): 155-164

\section{GENERAL CONSIDERATIONS}

A general consideration is that several forms of primitive and systemic vasculitis, regardless of the size of the affected vessel, may exhibit identical histological alterations. Table I clarifies this concept with the example of giant cells, which are certainly present in the arteritis with the same name but which can also be found in biopsies of arteries affected by Takayasu's arteritis (TA) or by granulomatosis with polyangiitis (formerly Wegener's granulomatosis).

This observation has important clinical implications as it means that cases of vasculitis may not correspond clinically and histologically. Thus, while histology remains the diagnostic gold standard, it can be used only as part of the most complete clinical assessment possible.

Other considerations include the fact that histological lesions of vasculitis, may also be detected in other conditions, such as infections, neoplasms, drug reactions, or vasculopathies. Among these are tuberculosis, mycotic aneurysms, pulmonary hypertension, malignant hypertension, cocaine abuse, atrial myxoma,
Table I - Primitive and systemic vasculitis, regardless of the size of the affected vessel, may exhibit identical histological alterations. GPA, granulomatosis with polyangiitis; AAV, ANCA-associated vasculitis; HBV, hepatitis B virus-related vasculitis; IP, malignant hypertension; EGPA, eosinophilic granulomatosis with polyangiitis (From Dr Cavazza A. personal communication).

\begin{tabular}{|l|l|l|}
\hline & $\begin{array}{c}\text { Histopathological } \\
\text { lesion }\end{array}$ & \multicolumn{1}{|c|}{$\begin{array}{c}\text { Disease } \\
\text { entity }\end{array}$} \\
\hline & Giant cells & $\begin{array}{l}\text { - Giant cells } \\
\text { arteritis } \\
\text { - Takayasu's } \\
\text { arteritis } \\
\text { - GPA }\end{array}$ \\
\hline & Fibrinoid necrosis & $\begin{array}{l}\text { - Panarteritis } \\
\text { nodosa } \\
\text { - AAV } \\
\text { - HBV, IP etc. }\end{array}$ \\
\hline & $\begin{array}{l}\text { Eosinophil } \\
\text { granulocytes }\end{array}$ & $\begin{array}{l}\text { EGPA } \\
\text { - Allergic } \\
\text { vasculitis } \\
\text { - Giant cells } \\
\text { arteritis }\end{array}$ \\
\hline & & GPA \\
\hline
\end{tabular}

Corresponding author: Gianluigi Bajocchi UOC di Reumatologia, Arcispedale S. Maria Nuova, IRCCS Reggio Emilia, Italy E-mail: gianluigi.bajocchi@ausl.re.it 
segmental arterial mediolysis and fibromuscular dysplasia (1-6).

A further point is that vasculitis histology changes over time, as do disease evolution and activity, even without considering the masking effects of treatment and the possibility of sampling error due to patchy localization that often characterize vasculitis. Again, it is evident that the clinical assessment of the disease is necessary in order to decide the most appropriate time and site of the biopsy. It includes the evaluation of treatmend dose and duration in order to avoid an unspecific or falsely negative histology.

In this regard, the size of the biopsy specimen should be also taken into account. Sampling errors are not unusual, as a consequence of the patchy involvement that often characterizes vasculitis, in which large sections of vessel or tissue areas are spared (1).

Lastly, the possibility of overlapping forms of vasculitis must be considered; this overlap can be detected both clinically and histologically $(7,8)$. In some occasions, a diagnosis of overlapping vasculitides may be the result of an incorrect interpretation of their physiopathology and of nomenclature. A careful reading of the 2012 Revised International Chapel Hill Consensus Conference Nomenclature of Vasculitides (9) reveals the key concept that each of the three categories of vasculitis (large, medium, small) may also include arteries of different sizes "...a key concept is that vasculitis of all 3 major categories can affect any size artery".

Indeed, the subdivision is set according to the higher frequency with which one of the three anatomical categories is involved in a specific vasculitis. Thus the presence of vessels of different sizes in a specific case of vasculitis does not necessarily imply overlap. Consider the involvement of the small ciliary arterioles causing the anterior ischemic optic neuritis (A.I.O.N.) that complicates giant cell arteritis (GCA). Concerning vasculitis of large vessels (LVV), the cited Consensus Conference nomenclature of vasculitides states (9): "CHCC 2012 defines LVV as vasculitis affecting the aorta and its major branches more often than other vasculitides, however, any size artery may be affected. This definition does not state that LVV predominantly affects large vessels because in many patients the number of medium and small arteries affected is greater than the number of large arteries affected". This type of imperfect nomenclature is a compromise made necessary by the lack of knowledge of the etiology for primitive vasculitis, which is therefore not usable for classification purposes.

\section{ANATOMICAL CONSIDERATIONS}

Anatomical outlines are necessary to understand the various histopathological pictures that involve the vessel walls (10). Indicatively, large vessels have a diameter of between $3 \mathrm{~cm}$ and $7 \mathrm{~mm}$, medium-sized vessels of between $7 \mathrm{~mm}$ and $2.5 \mathrm{~mm}$, and small vessels have a diameter under 2.5 $\mathrm{mm}$. Some texts consider small vessels those that have a diameter under $100 \mathrm{mi}$ crons $(0.1 \mathrm{~mm})$.

In the arteries and veins there are 3 tunicae and 2 elastic membranes, internal and external, which border with the tunicae but are also present with interruptions (fenestrations) in the tunica media thickness itself. Concentrically from the lumen are the tunica intima, the internal elastic membrane, the tunica media, the external elastic membrane, and the external tunica (adventitia). The tunica adventitia differs for its less compact structure and the presence of vasa vasorum in the most external part.

The veins have the same three-layer structure as the arteries though it is less well defined. The tunica media, and sometimes the external tunica, contain muscle fibres and strong elastic fibres, as well as connective tissue. To contrast the effects of hydrostatic overload, the amount of muscle fibre in the veins of the lower body is greater than that in the veins of the upper body. The adventitia is often not clearly demarcated as it tends to blend into the surrounding connective tissue.

Arterioles (pre-capillaries) are arteries with a diameter of less than $0.5 \mathrm{~mm}(500 \mathrm{mi}-$ crons). They have the same triple concentric structure of the arteries. Along with the 
capillaries and the post-capillary venules, they make up the microcirculation. Both elastic membranes are often absent in these vessels. The tunica media is composed of a layer of muscle cells with scant collagen fibres, while the adventitia is thin and composed of collagen fibres in connective tissue. In the arteries and arterioles, eccentric intimal thickening is the initial site that predisposes to the formation of calcified atherosclerotic plaques. This distinction between eccentric and diffuse can be exploited in the differential diagnosis between arteriosclerosis and inflammatory vasculitis.

Capillaries have a diameter of between 4 and 15 microns, thereby enabling the passage of a monolayer of red blood cells with a diameter of 7.2-7.9 microns. They regulate metabolic and oxygen exchange, favoured by the slow flow velocity. Capillary walls are made up of one or two layers of endothelial cells that are sometimes fenestrated (for example visceral splenic organs) placed on the basal membrane whose exterior is part of the perivascular matrix of pericytes. Hydrostatic pressure in the capillary bed is very low. This low pressure implies that bleeding in the capillary bed may not be detected for a long time, unless a very large anatomical area is involved such as in diffuse alveolar haemorrhage.

It is worth concluding this anatomical review by recalling the interpretation of the anatomical categories of the Revised International Chapel Hill Consensus Conference Nomenclature of Vasculitis (9), useful for the taxonomy of non-infectious vasculitis. In this work, large vessels are referred to as the whole aorta, the major epiaortic and subdiaphragmatic efferents and the corresponding veins, medium vessels are the arteries and veins and the main efferents, and for small vessels, the intraparenchymal arteries, capillaries and venules, i.e., the microcirculation.

\section{LARGE VESSEL VASCULITIDES (LVV) HISTOPATHOLOGY}

The most significant forms of vasculitis in clinical practice are giant cell arteritis, ju- venile temporal arteritis, Takayasu's arteritis, and chronic periaortitis (also defined as aortitis, such as IgG4-related or infectious). These forms may extend to the peritoneum, thereby determining a clinical picture of retroperitoneal fibrosis.

\section{GIANT CELL ARTERITIS (GCA)}

Preliminary studies in the 1990s, as well as confirmation from recent large series of cases, have shown that the spectrum of GCA goes beyond the classic lesion, which presents a transmural inflammatory infiltrate with variable presence of granulomas containing also multinucleate giant cells, associated with the fragmentation of the internal and external elastic membrane and proliferation of the intima.

A review of 354 positive biopsies of temporal arteries (11) showed that 274 cases (77.5\%) had the classic transmural inflammation (TMI) that crosses the external elastic membrane, extending to the tunica media. In the remaining $22.5 \%$ with clinical diagnosis of GCA 3 types of lesions were seen in which the temporal artery wall was apparently spared (Figure 1). Three patterns were seen in very similar percentages: GCA with small vessel vasculitis (SVV) of the small periadventitial vessels lacking muscle tunica (32 cases, $9 \%$ ); vasa vasorum vasculitis (VVV), in which inflammation affects only the intraadventitial vasa vasorum (23 cases, $6.5 \%$ ); an intermediate picture in which a limited number of inflammatory infiltrates were present only in in the adventitia, inflammation limited to adventitia (ILA) but with a distribution both perivascular and throughout the adventitial tissue ( 25 cases, $7 \%$ ). In this group of GCA with arterial wall sparing, patients with SVV and VVV had lower mean acute inflammation reactants, fewer cranial symptoms (headache, jaw claudication), paucity of temporal artery objectivity, and lower doses of steroids at the time of biopsy. Thus, in theory, these are patients with uncertain clinical diagnosis but who have the same risk of blindness as those with classic biopsy features (TMI pattern). 
It is interesting to note that this observation can be explained only with inflammation originating in the adventitia, the periadventitial capillaries and from the intra-adventitial vasa vasorum. This concept is reinforced by the observation of the group of biopsies with classic TMI, which reveals that there are more inflammatory infiltrates farther from the lumen and thus in the layer between the adventitia and the tunica media rather than between the tunica media and the intima. Lastly, further confirmation of an inflammatory trigger from the microvascular adventitia is demonstrated by the increased expression in the endothelium of Jagged1, a protein recognized by CD4+ that induces Th1 and Th17 polarization, which have autoimmune and inflammatory features (Figure 1) (12).

The pathogenic importance of the isolated inflammation of vasa vasorum was initially pointed out by Disdier (13). His work on a sample of 28 patients found vasculitis of the adventitial vasa vasorum (vasa vasorum pattern according to Cavazza) of the temporal artery in 5 patients with panarteritis nodosa, confirmed by the presence of necrotizing vasculitis in muscle biopsy, polymyalgia rheumatica in 4 patients without cranial symptoms, and, in a mix of 17 other cases, including a relapsing polychondritis, a glomerulonephritis with crescentic lesions, two cases of cryoglobulinemia (associated with polyangiitis with granu-

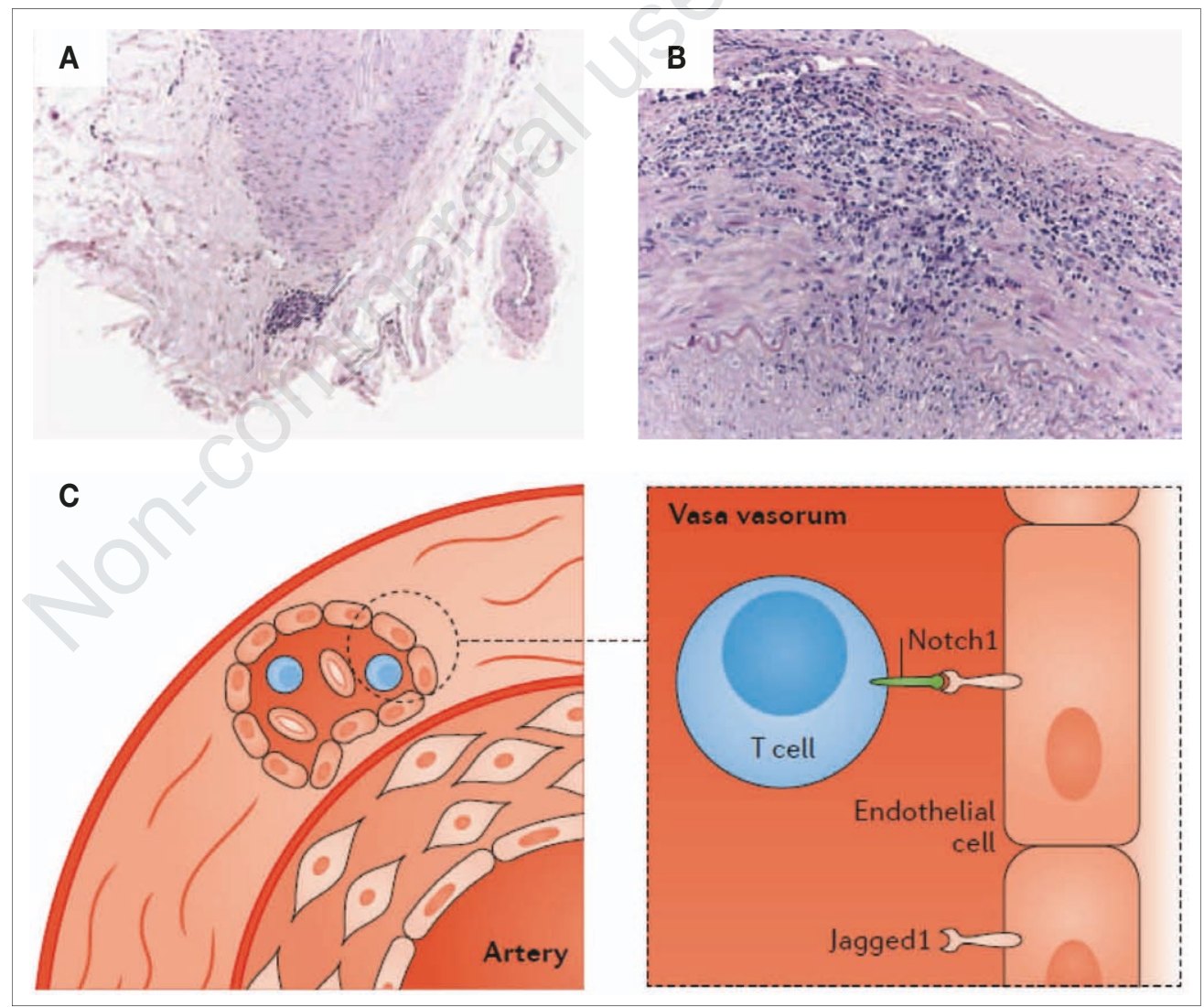

Figure 1 - Biopsies of arteries of patients with GCA. Inflammatory infiltrate with vasa vasorum vasculitis (VV) vasa vasorum vasculitis (A) and transmural inflammation (TMI) (B) patterns (see text) and Notch signalling pathway in the vasa vasorum of adventitia. A) Pattern VW, in which inflammation was limited to the adventitial vasa vasorum; B) pattern TMI, inflammatory cells penetrate through the external elastic lamina and extend to the media (from Cavazza A.) C) Engagement of endothelial Jagged1 triggers Notch signalling in CD4+ T cells and biases them towards differentiating into pro-inflammatory TH1 and TH17 cells (modified from Collison $\mathrm{J}$ and Cavazza A). 
lomatosis and with HCV infection, respectively), a case of rheumatoid arthritis, and some neoplasms. Even in this case it should be noted that the inflammatory infiltrate of the adventitia is not exclusive of LVV thus recalling the concept that clinical findings and histology may possibly not agree.

In the cases in Cavazza's study (11), temporal artery inflammation with a classical TMI lesion was present in a patient with panarteritis nodosa; a VVV type lesion was present in three cases of ANCA-positive small vessel vasculitis, and a GCA inflammation with ILA-type features was present in a patient with primary amyloidosis vasculitis. Muratore's (14) and Cox's (15) studies analyzed the various lesions using histological slides that were able to identify GCA in remission or, in the case of negative biopsies, an arteriosclerotic vasculopathy, and found that transmural inflammatory infiltrates are the only useful lesions for differential diagnosis.

The Salvarani group has shown that negative temporal artery biopsies of subjects with clinical diagnosis of GCA are quite similar to those of subjects of similar age but without GCA.

Thus, only the transmural inflammatory infiltrate, and none of the histological alterations studied (middle-intimal scars, adventitious fibrosis with angiogenesis, calcifications, intimal hyperplasia and fragmentation of the internal elastic lamina), are useful for differential diagnosis between GCA and vasculosclerosis. Cox (15), analyzing the histological lesions useful to distinguish between remission and arteriosclerotic vasculopathy, reached the same conclusion: the transmural inflammatory infiltrate is the only criterion useful for differential diagnosis between the two vascular forms.

\section{JUVENILE TEMPORAL ARTERITIS (JTA)}

Involvement of temporal artery before the age of 40 can be considered juvenile temporal arteritis (JTA) (16). Histologically, severity ranges, from simple transmural eosinophilic inflammatory infiltrates to le- sions related to classic GCA, with the destruction of the internal elastic membrane and marked hyperplasia of the intima. Arterial thrombosis is sometimes present. The presence of giant cells is not part of JCA (Figure 2). Based more on histological than clinical findings (headache), the differential diagnosis of a JTA is therefore:

1) a very rare juvenile form (between 20 to 30 years of age) of the GCA spectrum; 2) a temporal arteritis secondary to a pol-

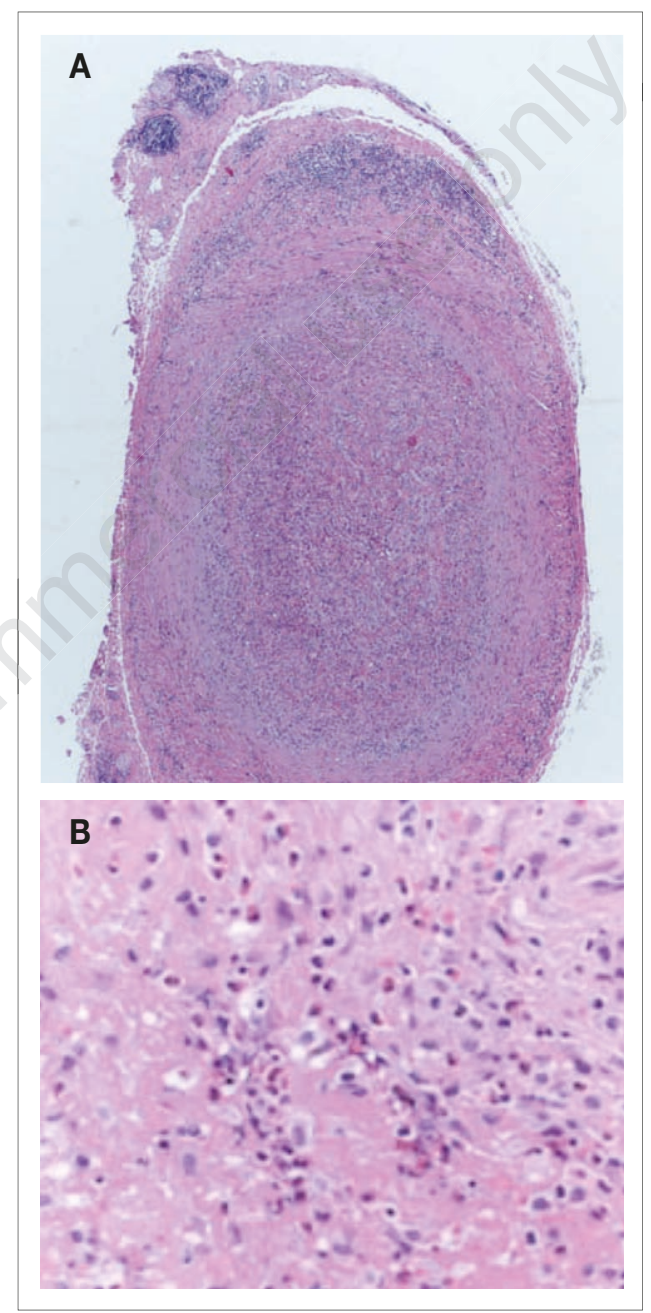

Figure 2 - A) Temporal artery biopsy showing an acute thrombosis with a transmural predominantly lymphocytic infiltrate. Hematoxylin-Eosin, 40X. B) Detail of the acute thrombosis, showing an eosinophilic infiltrate intermingled with fibrin. Hematoxylin-Eosin, 400X (Dr Caruso and Dr Cavazza, personal observation). 
yarteritis nodosa or an ANCA-associated vasculitis;

3) Kimura's disease;

4) angiolymphoid hyperplasia with eosinophilia (ALHE), for the presence of eosinophilic infiltrate with varying degrees of vascular proliferation (17).

In patients with JTA, the iexcision performed for histological diagnosis leads to a resolution of symptoms. The juvenile form of GCA requires steroid therapy.

\section{TAKAYASU'S ARTERITIS (TA)}

The histology of TA is consistent with that of GCA, and despite the fact that a larger inflammatory and necrotizing component (microabscesses), more giant cells, and more adventitial thickening have been reported, these elements are not in themselves sufficient to distinguish it from GCA in the absence of clinical and imaging support. Clinically it is worth noting the involvement of the elastic pulmonary arteries.

The latest histological works date back to the 1990s $(1,18)$. In acute phases, there are granulomatous lesions with transmural inflammation and skipping destruction of muscle-elastic lamina within the tunica

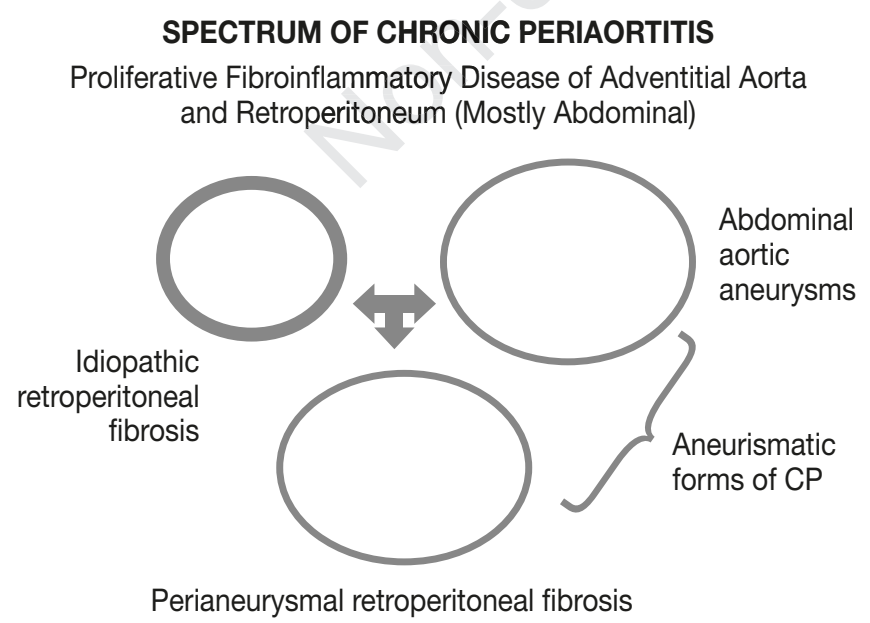

Figure 3 - Chronic periaortitis (CP) includes idiopathic retroperitoneal fibrosis, abdominal aortic aneurysms, and a combination of the two diseases called perianeurysmal retroperitoneal fibrosis (from Parums DV. The spectrum of chronic periaortitis. Histopathology 1990; 16 : 423-31). media, quite similar to GCA. The infiltrate is mainly lymphoplasmacytic within the media and with the presence of giant cells (both Langhans and foreign-body type). In the remission or healing phases, a progressive adventitial and intimal fibrosis with extended scarring of the media occur. The infiltrate is minimal and lymphocytic.

\section{CHRONIC PERIAORTITIS (CP) AND RETROPERITONEAL FIBROSIS}

The classification of chronic periaortitis (CP) dates back to Mitchinson in 1984 (19) and is composed of three anatomopathological forms where perianeurismatic retroperitoneal fibrosis is likely the combination of the first two (Figure 3). The inflammatory aneurysms of large vessels account for about $10 \%$ of those that are usually based on arteriosclerosis. Histology reveals a vasculitis that involves the vasa vasorum of the aortic wall but extends to the retroperitoneal vessels in an anatomical continuum between the two structures of the aortic adventitia and the peritoneal folds (20). Although large studies are scanty, CP seems to be characterized by a greater number of collagen fibres compared to large vessel vasculitis. The adventitia appears to be the preferential site of chronic inflammation, resulting in fibrosis and thickening which surrounds the large vessels by creating a sleeve that stenizes the vessel. For diagnosing IgG4-related systemic disease (21), at least $50 \%$ of lymphoplasmacytic infiltrate of the adventitia must be IgG4-positive (Figure 4). Fibrinoid necrosis is sometimes noted in the adventitial vessels of CP, which is the marker of this vasculitis. Cases of association with vasculitis have been described, especially of granulomatosis with polyangiitis (GPA), and urticarial vasculitis (22). Lastly, the involvement of the adventitial vessels in $\mathrm{CP}$ associates with GCA with involvement of the small adventitial vessels in $23 \%$ of cases (11). All these elements, therefore, suggest including $\mathrm{CP}$ in the field of vasculitis (aortitis) and retroperitoneal fibrosis in a pathological continuum. It is very likely that retroperitoneal fibrosis (sclerosing mediastinitis, inflammatory an- 
eurysms of the aorta) is in fact an extension of chronic periaortitis. The macroscopic appearance of retroperitoneal fibrosis is broad in range: dark yellow, white-grey, firm to gelatinous. Histologically, the appearance may vary from myxomatous tissue to abundance of elongated elliptical cells, so-called spindle cells, such as those present in sarcoidosis or some neoplasms, to an appearance of dense acellular collagen. Initially, an infiltrate of lymphocytes, plasma cells and macrophages is often described. Typically, necrosis and granulomas are absent.

\section{SMALL VESSEL VASCULITIS (SVV)}

Small vascular vasculitis (SVV) is subdivided into AAV (anti-neutrophil cytoplasm antibody associated vasculitis) and SVV (immune complex small vessel vasculitis). The former include granulomatosis with polyangiitis (GPA), formerly Wegener's granulomatosis, eosinophilic GPA (EGPA), formerly Churg-Strauss, and microscopic polyangiitis (MPA) $(1,9)$. These diseases are characterized by:

1) presence of ANCA antibodies;

2) fibrinoid necrosis of the media;

3) minimal presence of immune complexes/antibodies (pauci-immune).

All three forms may extend, though not often, to medium-sized vessels. Fibrinoid necrosis is the pathognomonic lesion of acute or active vasculitis (23), and is the product of endothelial breakage that allows plasma to filter into the extra-vascular space and vascular wall, triggering a coagulation cascade with subsequent final production of fibrin filaments.

Initial endothelial damage is the consequence of the infiltrate of neutrophils and monocytes, which are sensitized by contact with cytokines and fraction $\mathrm{C} 5 \mathrm{a}$, and are then activated by binding to ANCA (22). Extravascular necrotic granulomas are only present in the two granulomatous forms (GPA and EGPA). Granulomas are the reaction after necrosis. The minimum definition of granuloma is an aggregate of histiocytes (macrophages) regardless of whether

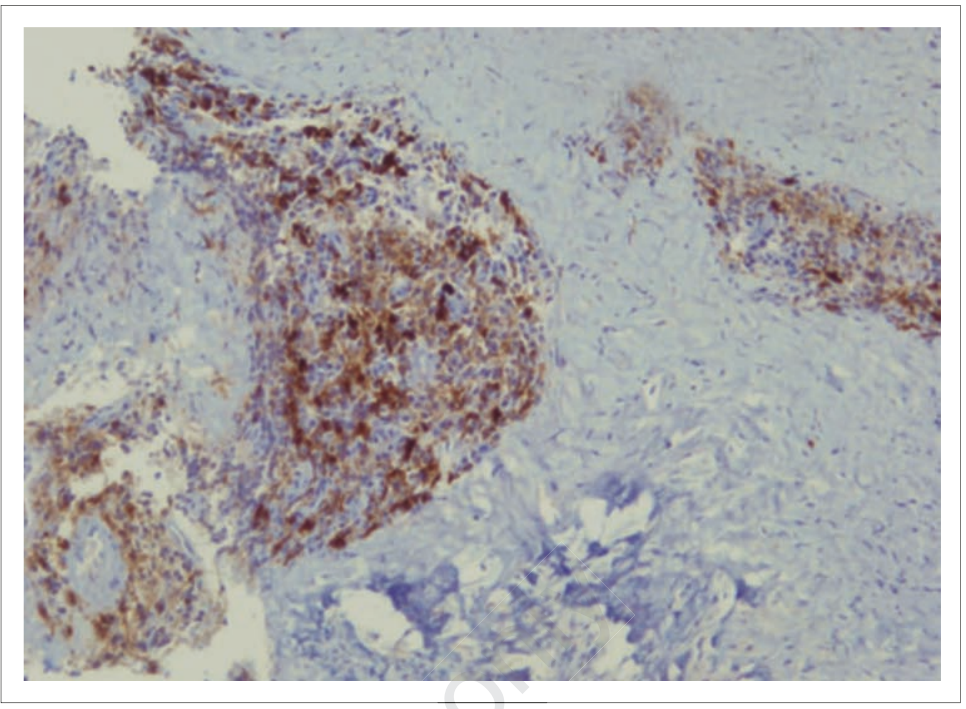

Figure 4 - Chronic periaortitis within a related lgG4 syndrome. Abdominal aortic wall adventitia fragments extensively replaced by fibrous hyaline tissue with large collagen bands and diffuse perivascular inflammatory infiltrate. Significant presence of IgG4 + plasma cells (personal observation).

the lesion contains necrosis, lymphocytes, plasma cells or multinucleated cells (23). Sometimes for their elongated appearance and undefined margins.

It is worth remembering that biopsy may not be necessary for diagnosis. Indeed, ANCA positivity is present in $70-90 \%$ of active, diffuse, GPA and MPA with pulmonary and renal localizations. Biopsy may, on the other hand, be necessary in the sinus localization where the percentage of ANCA positivity is minimal. The presence of ANCA is documented only in $40-50 \%$ of the limited forms of GPA and MPA and in $30-40 \%$ in EGPA (24).

Extravascular eosinophil infiltrate and eosinophilia by at least $10 \%$ are ACR criteria in EGPA (25). A moderate infiltrate is also present in GPA and in the vasculitis of medium vessels (Kawasaki in 22-36\% of cases), while it is generally absent or scarce in large vessels vasculitis (26). In other cases, the presence of eosinophils is the marker of a variety of diseases such as asthma, intestinal parasites, drug reaction or hypereosinophilic syndrome.

Eosinophils infiltrating the vascular wall can cause direct cytotoxic damage through 
the release of eosinophilic proteins and oxygen-reactive molecules, or indirectly, as a result of recruitment and activation of other inflammatory cells. Their activation also triggers tissue fibrosis, thrombotic diathesis (hypercoagulability) and allergic inflammation. Which of these three processes is more pathogenetic depends on the tissue involved (26).

In EGPA, after the first stage characterized by asthma, chronic rhinosinusitis and/ or nasal polyposis, a second phase of eosinophilia in the circulation and tissues will follow. Ultimately, an eosinophilic vasculitis of the small arteries and veins, with eosinophilic granulomas, will become evident. The structure of eosinophilic granulomas exhibit a central area of eosinophilic necrosis surrounded by histiocytes and polynuclear giant cells. The involvement of various vessels, small arteries and veins depends on the stage of the disease (26). The other (predominantly) small forms of vasculitis are immune complex related (SVV). These includes IgA vasculitis (IgAV, formerly Henoch-Schönlein purpura), cryoglobulinemic vasculitis (HCV or lymphoblastic B-related), Goodpasture syndrome (anti-membrane antibodies) and hypocomplementemic urticarial vasculitis (associated with anti-C1q antibodies). In these pathologies, arterial involvement is much rarer than in ANCA group. (27).

They are commonly associated with:

1) frequent presence of autoantibodies ( $\operatorname{Ig} \mathrm{A})$ or circulating immune-complexes that can bind the complement found in granular or linear deposits along the basal membranes of the tissue or in perivascular precipitates;

2) presence of hypersensitivity vasculitis or leukocytoclastic inflammation (nuclear fragmentation) more often clinically expressed in a palpable purpura (extravascular vascular extravasation) or macular papular skin rash.

The parietal inflammatory infiltrate is neutrophilic, while the eosinophilic one can also be present and diffused through the extravascular space (27). There may be varying degrees of endothelial interruption, endothelial cell hypertrophy in post- capillary veins, bleeding with intravascular fibrin thrombosis and fibrinoid necrosis. It is interesting to note that approximately $30-40 \%$ of cases of IgG4 syndrome are associated with complement deficiency.

The third group of vasculitis of small vessels includes secondary forms of systemic diseases, although it is not entirely clear whether they are primitive and associated or manifestations of the underlying disease, systemic lupus erythematosus, Sjögren's syndrome, rheumatoid arthritis, sarcoidosis. The whole microcirculation may be affected: arterioles, capillaries, venules. It is important to note that sarcoidosis is a granulomatous disease with no evidence of necrosis.

\section{VASCULITIS OF THE MEDIUM VESSELS}

This includes panarteritis nodosa (PAN) and Kawasaki's disease.

PAN is characterized by the presence of fibrinoid necrosis, which involves in particular the medium and small arteries, sparing smaller vessels: arterioles, capillaries and venules. In addition to the ANCA negativity, the absence or extreme rareness of granulomatous lesions and pulmonary and glomerular capillaritis distinguishes it from granulomatous AAV and microscopic polyangiitis (MPA). The infiltrate is pleomorphic, typically transmural, focal or segmental in distribution and contributes to the formation of microaneurysms (1). Endoluminal thromboses are often found as sequelae of necrosis. The uniqueness of PAN is the coexistence of necrotizing active lesions and repair vessel segments characterized by fibrotic proliferation. Correlation with infectious agents (Streptococcus for skin and HBV for systemic form) is established. A very interesting observation is that several PAN patients have CECR1 mutations (cat eye syndrome chromosome region, candidate 1), determining ADA2 (adenosine deaminase 2) deficiency. ADA2 has been shown to have a protective effect on the endothelium and to suppress some proinflammatory macrophage phenotypes (28). 


\section{VARIOUS CALIBERS OF VASCULITIDES (VVV)}

Various calibre of vasculitis (VVV) cannot be defined for their predominant vessel size, for which any type (arteries, veins, capillaries) and vessel dimensions (small, medium, large) can be involved. In this group, besides the more well-known Behçet disease (BD), which we will discuss below, is Cogan's disease. Clinically, both forms can manifest with aneurysms.

In $\mathrm{BD}$, mucosal biopsies of initial lesions such as aphthosis, follicles, papules and pustules more often show a leukocytoclastic vasculitis or perivascular neutrophilic infiltrate, while the most advanced lesions are characterized by the presence of lymphocytic infiltration (29).

A frequent manifestation of BD mucosal form is erythema nodosum. Histologically, this is a panniculitis, more often lobular, sometimes mixed (septal and lobular) with an infiltrate of neutrophils, lymphocytes and histiocytes, interspersed with necrotic adipocytes.

Vasculitis with lymphocytic parietal infiltrate or leukocytoclastic vasculitis, sometimes with associated phlebitis and arteriolitis (30), are almost always present in the erythema nodosum of $\mathrm{BD}$, but not in the primitive erythema nodosum.

In the biopsy of positive regions at the pathergy test (skin hyperreactivity in response to minimal trauma), Indian authors documented a mixed inflammatory subcutaneous infiltrate, or neutrophil or lymphocytic predominance, and a lobular panniculitis without vasculitis (31).

\section{SINGLE ORGAN VASCULITIS (SOV)}

This is vasculitis manifested in a single organ and can affect vessels of any type and diameter (32). These forms have no specific features suggesting they are a limited expression of a systemic vasculitis. This group includes, for example, skin or intestinal panarteritis nodosa, involvement of small and medium vessels of the CNS, genital giant cell arteritis, isolated aortitis, etc.

\section{CONCLUSIONS}

In recent years, and starting from the 1994 International Chapel Hill Consensus Conference on the Nomenclature of Systemic Vasculitides (CHCC 1994), which proposed names and definitions for the most common forms of vasculitis, there have been substantial advances in our understanding of this class of diseases. The subsequent important advance on vasculitis was the second International CHCC in 2012, which modified names and definitions, and added important categories of vasculitis not included in CHCC 1994.

However, the relevance of the two events should not make us forget that what we use every day in clinical practice is an elegant nosographic structure (nomenclature) that remains far from any form of classification and even farther from any the diagnostic criteria. These criteria would require a knowledge of ethiology which we still lack for most forms of vasculitis apart from secondary ones. This concept must be kept in mind whenever we suspect a vasculitis.

A knowledge of ethiology and pathogenetic mechanisms would certainly have an impact on classification. Consider for example the success of ANCAs in identifying and correlating a precise subgroup of vasculitides. Or the benefits arising from the knowledge of the pathogenetic role of CD20 and IL-6, respectively for the vasculitides of small and large vessels, which has led us to developing a new generation of treatments that have exploited this knowledge.

The issue of vasculitis has a long way to go; there are several unmet needs regarding classification and markers of disease. However, much progress has been made and all the data on quality of and life expectancy for our patients confirm this.

\section{REFERENCES}

1. Lie JT. Illustrated histopathologic classification criteria for selected vasculitis syndromes. Arthrit Rheum. 1990; 33: 1074-87.

2. Jain A, Misra DP, Ramesh A, et al. Tuberculosis mimicking primary systemic vasculitis: not to be missed! Trop Doct. 2017; 47: 158-64.

3. de Havenon A, McNally S. Response to 
"High-Resolution Vessel Wall MRI: Appearance of Intravascular Lymphoma Mimics Central Nervous System Vasculitis". Clin Neuroradiol. 2016; 26: 501.

4. Mahr A, Batteux F, Tubiana S, et al Brief report: prevalence of antineutrophil cytoplasmic antibodies in infective endocarditis. Arthrit Rheumatol. 2014; 66: 1672-7.

5. Walsh NM, Green PJ, Burlingame RW, et al. Cocaine-related retiform purpura: evidence to incriminate the adulterant, levamisole. J Cutan Pathol. 2010; 37: 1212-9.

6. Baker-LePain JC, Stone DH, Mattis AN, et al. Clinical diagnosis of segmental arterial mediolysis: differentiation from vasculitis and other mimics. Arthritis Care Res. 2010; 62: 1655-60.

7. Nishino H, DeRemee RA, Rubino FA, et al. Wegener's granulomatosis associated with vasculitis of the temporal artery: report of five cases. Mayo Clin Proc 1993; 68: 115-21.

8. Chirinos JA, Corrales VF, Lichtstein DM. ANCA-associated large vessel compromise. Clin Rheumatol. 2005; 25: 111-2.

9. Jennette JC, Falk RJ, Bacon PA, et al. 2012 Revised International Chapel Hill Consensus Conference Nomenclature of Vasculitis. Arthritis Rheum. 2013; 65: 1-11.

10. Kuehnel W. Vessel Histology. In: Kuehnel W (ed.) Color Atlas of Cytology, Histology, and Microscopic Anatomy. Thieme 4th ed. 2003; 274-305.

11. Cavazza A, Muratore F, Boiardi L, et al. Inflamed temporal artery: histologic findings in 354 biopsies, with clinical correlations. Am J Surg Pathol. 2014; 38: 1360-70.

12. Wen Z, Shen Y, Berry G, et al. The microvascular niche instructs $T$ cells in large vessel vasculitis the VEGF-Jagged1-Notch pathway. Sci Transl Med. 2017; 9: 3322.

13. Disdier P, Pellissier JF, Harle JR, et al. Significance of isolated vasculitis of the vasa vasorum on temporal artery biopsy. J Rheumatol. 1994; 21: 258-60.

14. Muratore F, Cavazza A, Boiardi L. Histopathologic Findings of Patients With BiopsyNegative Giant Cell Arteritis Compared to Those Without Arteritis: A Population-Based Study. Arthritis Care Res. 2016; 68: 865-70.

15. Cox M, Gilks B. Healed or quiescent temporal arteritis versus senescent changes in temporal artery biopsy specimens. Pathology. 2001; 33: 163-6.

16. McGeoch L, Silecky WB, Maher J, et al. Temporal arteritis in the young. Joint Bone Spine. 2013; 80: 324-7.

17. Fukunaga M. Juvenile temporal arteritis associated with Kimura's disease. APMIS. 2005; 113: 379-84.
18. Lie JT. The classification and diagnosis of vasculitis in large and medium-sized blood vessels. Pathol Annu. 1987; 22: 125-62.

19. Mitchinson MJ. Chronic periaortitis and periarteritis Histopathology. 1984; 8: 589-600.

20. Kamisawa T, Zen Y, Pillai S, et al. IgG4-related disease. Lancet. 2015; 385: 1460-71.

21. Yamamoto M, Takahashi H, Shinomura Y. Mechanisms and assessment of IgG4-related disease: lessons for the rheumatologist. Nature Rev Rheumatol. 2014; 10: 148-59.

22. Takao M, Hamada T, Kaji T, et al. Hypocomplementemic urticarial vasculitis arising in a patient with immunoglobulin G4-related disease. Int J Dermatol. 2016; 55: 430-3.

23. Jennette JC, Falk RJ. Pathogenesis of antineutrophil cytoplasmic autoantibody-mediated disease. Nat Rev Rheumatol. 2014; 10: 463-73.

24. Hagen EC, Daha MR, Andrassy K, et al. For the EC/BCR Project for ANCA assay standardization: diagnostic value of standardized assays for anti-neutrophil cytoplasmic antibodies in idiopathic systemic vasculitis. Kidney Int. 1998; 53: 743-53.

25. Masi AT, Hunder GG, Lie JT. The American College of Rheumatology 1990 criteria for the classification of churg-strauss syndrome (allergic granulomatosis and angiitis). Arthritis Rheum. 1990; 33: 1094-100.

26. Khoury P, Grayson PC, Klion AD. Eosinophils in vasculitis: characteristics and roles in pathogenesis. Nat Rev Rheumatol. 2014; 10: 474-83.

27. Calabrese LH, Michel BA, Bloch DA, et al. The American College of Rheumatology 1990 criteria for the classification of hypersensitivity vasculitis. Arthrit Rheum. 1990; 33: 1108-13.

28. Navon Elkan P, Pierce SB, Segel R, et al. Mutant adenosine deaminase 2 in a polyarteritis nodosa vasculopathy. N Engl J Med. 2014; 370: 921-31.

29. Rios Scherrer MA, Barreto Rocha V, Campos GL. Behecet's disease: review with emphasis on dermatologica aspects. Ann Bras Dermatol. 2017; 92: 452-64.

30. Kim B, LeBoit PE. Histopathologic features of erythema nodosum-like lesions in Behçet disease: a comparison with erythema nodosum focusing on the role of vasculitis. Am J Dermatopathol. 2000; 22: 379-90.

31. Ekin O, Ilknur B, Ozlem A. Histopathologic study of pathergy test in Behçet's disease. Indian J Dermatol. 2014; 59: 630.

32. Loricera J, Blanco R, Ortiz-Sanjuán F, Singleorgan cutaneous small-vessel vasculitis according to the 2012 revised International Chapel Hill Consensus Conference Nomenclature of Vasculitides: a study of 60 patients from a series of 766 cutaneous vasculitis cases. Rheumatology (Oxford). 2015; 54: 77-82. 